\title{
Procoagulatory changes induced by head- up tilt test in patients with syncope: observational study
}

\author{
Viktor Hamrefors ${ }^{1,2,3} \mathbb{D}$, Artur Fedorowski ${ }^{1,4^{*}+}$, Karin Strandberg ${ }^{5}$, Richard Sutton ${ }^{6}$ and Nazim Isma ${ }^{1,7 \dagger}$
}

\begin{abstract}
Background: Orthostatic hypercoagulability is proposed as a mechanism promoting cardiovascular and thromboembolic events after awakening and during prolonged orthostasis.

We evaluated early changes in coagulation biomarkers induced by tilt testing among patients investigated for suspected syncope, aiming to test the hypothesis that orthostatic challenge evokes procoagulatory changes to a different degree according to diagnosis.

Methods: One-hundred-and-seventy-eight consecutive patients (age, $51 \pm 21$ years; $46 \%$ men) were analysed. Blood samples were collected during supine rest and after 3 min of $70^{\circ}$ head-up tilt test (HUT) for determination of fibrinogen, von Willebrand factor antigen (VWF:Ag) and activity (VWF:GP1bA), factor VIII (FVIII:C), lupus anticoagulant (LA1), functional APC-resistance, and activated prothrombin time (APTT) with and without activated protein C (C+/-). Analyses were stratified according to age, sex and diagnosis.

Results: After 3 min in the upright position, VWF:Ag $(1.28 \pm 0.55$ vs. $1.22 \pm 0.54 ; p<0.001)$ and fibrinogen $(2.84 \pm 0.60$ vs. $2.75 \pm 0.60, p<0.001)$ increased, whereas APTT/C+/- (75.1 \pm 18.8 vs. $84.3 \pm 19.6 \mathrm{~s} ; p<0.001$, and $30.8 \pm 3.7$ vs. $32.1 \pm 3.8$ s; $p<0.001$, respectively) and APC-resistance ( $2.42 \pm 0.43$ vs. $2.60 \pm 0.41, p<0.001)$ decreased compared with supine values. Significant changes in fibrinogen were restricted to women $(p<0.001)$ who also had lower LA1 during HUT ( $p=0.007$ ), indicating increased coagulability. Diagnosis vasovagal syncope was associated with less increase in VWF:Ag during HUT compared to other diagnoses (0.01 \pm 0.16 vs. $0.09 \pm 0.17 ; p=0.004)$.

Conclusions: Procoagulatory changes in haemostatic plasma components are observed early during orthostasis in patients with history of syncope, irrespective of syncope aetiology. These findings may contribute to the understanding of orthostatic hypercoagulability and chronobiology of cardiovascular disease.
\end{abstract}

Keywords: Orthostatic stress, Hypercoagulability, Coagulation factors, Partial thromboplastin time, Fibrinogen, von Willebrand factor

\section{Background}

It has long been observed that major cardiovascular and thromboembolic events show higher incidence after awakening, which has been partly explained by increased platelet activity during morning hours [1-4]. In parallel, a phenomenon of "orthostatic hypercoagulability", i.e.

\footnotetext{
* Correspondence: Artur.fedorowski@med.lu.se

${ }^{\dagger}$ Equal contributors

'Department of Clinical Sciences Malmö, Lund University, SE 205-02 Malmö, Sweden

${ }^{4}$ Department of Cardiology, Skåne University Hospital, Inga Marie Nilssons gata 46, SE 205-02 Malmö, Sweden

Full list of author information is available at the end of the article
}

increase in plasma procoagulants independent of postural volume shift and extravascular fluid escape, has been reported [5-7]. The prothrombotic changes during passive orthostasis were first detected in healthy volunteers without history of syncope, cardiovascular or thromboembolic disease. Similar changes in coagulation factors were induced by lower-body negative pressure in volunteers who developed vasovagal reflex but, interestingly, those who did not develop the reflex showed no significant alterations in coagulation factors [8]. Changes in the coagulation system have also been detected during vasovagal reflex activation: a study 
performed in subjects with von Willebrand disease has demonstrated increased antigen concentration of von Willebrand factor (VWF:Ag), VWF-Ristocetin-cofactor, and factor VIII activity (FVIII:C) after fainting prompted by fear of venepuncture [9]. In a previous study, we have observed that von Willebrand factor is increased in patients with orthostatic hypotension compared with other patients investigated for suspected syncope, irrespective of body position [10]. However, we have also noticed that both groups demonstrated procoagulatory changes during passive orthostasis evoked by head-up tilt test (HUT). In this study, we took the opportunity to analyse plasma samples collected at rest and during the early phase of HUT to assess how passive orthostasis impacts coagulation factors. We aimed to test the hypothesis that passive orthostasis during HUT would evoke procoagulatory changes and that these changes would differ according to HUT diagnosis.

\section{Methods}

\section{Patients and inclusion/exclusion criteria}

The present study was performed from November 2011 to October 2012 as a part of the ongoing Syncope Study of Unselected Population in Malmo (SYSTEMA) project, a single-centre observational study on syncope aetiology [11]. During this period, 233 consecutive patients underwent head-up tilt test (HUT) due to unexplained syncope and/or orthostatic intolerance. Patients were included if they accepted blood sampling during HUT ( $n=183$ ), and excluded if they were on current oral anti-coagulation therapy with warfarin $(n=5)$, leaving 178 patients that were eligible for the final inclusion. None of the included patients had a diagnosed bleeding disorder. All patients provided written informed consent and were included in further analyses. The Regional Ethical Review Board of Lund University approved the study protocol including blood sampling during tilt testing.

\section{Head-up tilt test}

Study participants were requested to take their regular medication and fast for $2 \mathrm{~h}$ before the test, although they were allowed to drink water. The examination was based on a specially designed HUT protocol, which included peripheral vein cannulation, supine rest for $15 \mathrm{~min}$ and HUT with optional nitroglycerine provocation after 20 min of passive HUT according to the Italian protocol [12]. Beat-to-beat blood pressure (BP) and ECG were continuously recorded using a Nexfin monitor (BMEYE, Amsterdam, The Netherlands) [13]. The detailed description of the examination protocol has been previously published [14]. The final HUT diagnoses were concordant with the current European Society of Cardiology guidelines [15].

\section{Blood sampling}

Blood was collected during supine rest before and after 3 min of $70^{\circ}$ HUT (during passive phase, prior to optional nitroglycerine administration) in citrated tubes (BD Vacutainer , 4,5 mL, 0,109 M sodium citrate). Samples were immediately centrifuged for $20 \mathrm{~min}$ at $2000 \mathrm{x}$ g. Plasma was separated and immediately frozen at $-70{ }^{\circ} \mathrm{C}$.

\section{Coagulation analyses}

APTT was performed at the accredited hospital laboratory using a BCS-XP analyzer (Siemens Healthcare, Marburg, Germany) with the Actin FSL reagents (Siemens Healthcare, Marburg, Germany), with and without addition of activated protein $\mathrm{C}$ (reference interval in healthy individuals for test without activated protein $\mathrm{C}$, 26-33 s). The analysis of plasma fibrinogen concentration was performed using the Dade Thrombin reagent on a Sysmex CS-5100 analyser (Siemens). The reference interval has been established locally as $2-4 \mathrm{~g} / \mathrm{L}$. The coagulation analyses below were performed on a BCS-XP instrument (Siemens). The original plasma-based activated protein $\mathrm{C}$ resistance (APC-resistance) test was performed with COATEST APC Resistance test (Chromogenix, Mölndal, Sweden). Reference interval was $>2.1$. The activity of von Willebrand factor (VWF:GP1bA) was analysed with an immunoassay based on an antibody against glycoprotein 1b (GP1b) and a GP1b construct that binds VWF in the sample (Innovance VWF:Ac; Siemens). Reference interval established locally was $0,5-2.0 \mathrm{kU} / \mathrm{L}$. Antigen concentration of von Willebrand factor (VWF:Ag) was measured with a latexenhanced immunoassays (Siemens). Reference interval was $0.6-2.7 \mathrm{kIU} / \mathrm{L}$. Screening test for lupus anticoagulant (LA1) was performed with a clot-based assay: LA1 (Siemens). Reference interval was $<42 \mathrm{~s}$. Factor VIII activity assay (FVIII:C) was performed with a chromogenic substrate method (Coatest FVIII SP, Chromogenic). Reference interval was $0.5-2.0 \mathrm{kIU} / \mathrm{L}$. Imprecision measured as a coefficient of variation was $2 \%$ for VWF:Ag (at $1.2 \mathrm{kIU} / \mathrm{L}$ ), 3\% for VWF:GP1bA (at $0.9 \mathrm{kIU} / \mathrm{L}$ ), $4 \%$ for LA1: (at $40 \mathrm{~s}$ ), 3\% for FVIII: (at 0.8 and $1.5 \mathrm{kIU} / \mathrm{L}$ ), and $2-4 \%$ for fibrinogen (at $1 \mathrm{~g} / \mathrm{L}, 2 \%$; at $3 \mathrm{~g} / \mathrm{L}, 4 \%$ ).

\section{Statistical analyses}

Independent samples Student's t-test was used to compare continuous variables between men and women whereas paired samples Student's $t$-test was used to compare changes in coagulation parameters measured in supine position and during HUT, respectively. Analyses regarding coagulation parameters were stratified according to sex and age (younger vs. older adults; 65 years). Changes in coagulation parameters during HUT according to diagnosis were specifically tested by comparing the changes in subjects with VVS versus all other 
diagnoses (including negative HUT) and comparing the changes in subjects with $\mathrm{OH}$ (classical + delayed form) versus all other diagnoses, using independent samples Student's t-test. Categorical variables were compared using Pearson's chi-square test. All analyses were performed using IBM SPSS Statistics version 23 (SPSS Inc., Chicago, IL, USA). All tests were two-sided whereby $p<0.05$ was considered statistically significant.

\section{Results}

Patient characteristics stratified by gender are shown in Table 1. Men were older and had lower resting heart rate. Sixty-nine $(38.8 \%)$ patients were diagnosed with vasovagal reflex syncope (VVS), whereas 49 (27.5\%) patients met $\mathrm{OH}$ criteria. Further, 7 patients had carotid sinus syndrome, 7 postural orthostatic tachycardia syndrome, 9 initial $\mathrm{OH}, 5$ psychogenic pseudosyncope and 17 (9.6\%) underwent HUT without a definitive diagnosis (i.e. negative test). Table 2 shows data on measurements of coagulation markers in supine and after $3 \mathrm{~min}$ of HUT. All patients had a significantly increased level of VWF:Ag and fibrinogen after 3 min of HUT, whereas APC-resistance ratio and APTT were significantly decreased compared with resting supine values. There were no significant differences between supine and HUT regarding FVIII, VWF:GP1bA and LA1. All parameters were within the reference interval for healthy individuals. In gender-stratified analyses, only women had significantly increased fibrinogen and demonstrated a shorter LA1 time during HUT (see: Table 3). Stratification of study population by the age of 65 years showed similar distribution of changes in coagulation parameters (Table 4). A final diagnosis of VVS was associated with

Table 1 Characteristics and risk factors in 178 consecutive patients with unexplained syncope, mean \pm SD or $n(\%)$

\begin{tabular}{lcccc}
\hline & All patients & Men & Women & $p$-value \\
& $n=178$ & $n=81(46)$ & $n=97(54)$ & \\
\hline Patient characteristics & & & & \\
Age (years) & $50.9 \pm 20.9$ & $56.0 \pm 18.7$ & $46.6 \pm 21.8$ & 0.003 \\
BMI (kg/ m2) & $25.6 \pm 4.6$ & $26.0 \pm 3.6$ & $25.3 \pm 5.3$ & 0.34 \\
Systolic BP (mmHg) & $131 \pm 18.7$ & $131 \pm 17.1$ & $132 \pm 20.0$ & 0.78 \\
Diastolic BP (mmHg) & $71 \pm 8.0$ & $71 \pm 8.8$ & $70 \pm 7.6$ & 0.44 \\
Heart rate (bpm) & $67.7 \pm 11.7$ & $65.4 \pm 12.7$ & $69.6 \pm 10.6$ & 0.016 \\
Risk factors (\%) & & & & \\
Hypertension & 28.8 & 35.0 & 23.7 & 0.10 \\
Diabetes mellitus & 6.7 & 8.6 & 5.2 & 0.36 \\
History of MACE & 3.9 & 7.4 & 1.0 & 0.029 \\
History of stroke & 1.7 & 2.5 & 1.0 & 0.46 \\
Present tobacco use & 15.7 & 16.0 & 15.5 & 0.92 \\
\hline
\end{tabular}

$\mathrm{BMI}$, body mass index; $\mathrm{BP}$, blood pressure; $\mathrm{MACE}^{\mathrm{a}}$ (=major coronary event) was defined as history of myocardial infarction, unstable angina, $\mathrm{CABG}$, or $\mathrm{PCI}$ less increase in VWF:Ag during orthostasis $(0.01 \pm 0.16)$ compared to all other diagnoses $(0.09 \pm 0.17 ; p=0.004)$. The changes in coagulation parameters were not different in patients diagnosed with $\mathrm{OH}$ compared with all other diagnoses $(p>0.05)$.

\section{Discussion}

The present study illustrates the impact of passive orthostatic challenge on haemostatic plasma components in a series of unselected patients with a history of unexplained syncope. During early phase of head-up tilt test, fibrinogen, APTT, VWF:Ag, and APC-resistance demonstrated significant procoagulatory changes. Although relative changes in the assessed coagulation markers were small, indeed, within the test imprecision range for fibrinogen, the directionality of these changes consequently pointed at the increased coagulability on standing.

Fibrinogen, as acute-phase glycoprotein, may be elevated in any form of inflammation and is also involved in formation of blood clots not only through fibrinformation, but also through its effect on specific platelet receptors and blood viscosity. Higher levels of fibrinogen are associated with cardiovascular disease [16-19] and its degradation products accumulate in the atherosclerotic plaque [20]. Interestingly, fibrinogen increased most in women who also demonstrated procoagulatory LA1 changes. The exact mechanisms behind these genderspecific propensities are not known and were not the subject of this study, however, hormonal differences may be one of the possible explanations [21]. Thus, orthostatic procoagulatory changes in fibrinogen, LA1, and FVIII seem mainly to affect women who are at higher risk of thromboembolic events compared with men [22]. As thromboembolic events are more common in the morning hours [3], orthostatic increase in fibrinogen and FVIII may act as a facilitator of thrombus generation after awakening and assuming an upright position, in a relative dehydrated state after a night's rest.

Notably, APTT was reduced on orthostasis irrespective of the analysed subgroup. Shortening of APTT usually indicates increased activity of FVIII in association with inflammatory conditions [23]. As APTT involves both fibrinogen and FVIII, which first and foremost increased in women, it seems that other factors such as prothrombin, V, IX, X, XI, and XII may have undergone orthostatic changes too. Unfortunately, our test panel did not detect this. Shortening of APTT, although within the normal range, may have thromboembolic consequences, as indicated by a previous study [24]. We believe that shortened APTT indicates a hypercoagulable state, as was also shown in a study by Mina et al. [25]. This is supported by other clinical studies performed on patients with acute coronary events [26, 27]. 
Table 2 Coagulation markers in supine and after 3 min head-up tilt (HUT) in 178 patients with unexplained syncope, mean \pm SD or n (\%)

\begin{tabular}{|c|c|c|c|c|c|}
\hline & All patients & $p$-value ${ }^{*}$ & Men & Women & $p$-valueq \\
\hline N (\%) & 178 & & $81(46)$ & $97(54)$ & \\
\hline FVIII:C supine (kIE/L) & $1.06 \pm 0.38$ & 0.43 & $1.07 \pm 0.32$ & $1.04 \pm 0.41$ & 0.56 \\
\hline FVIII:C HUT (kIE/L) & $1.07 \pm 0.36$ & & $1.07 \pm 0.35$ & $1.07 \pm 0.36$ & 0.98 \\
\hline WWF:Ag supine (kIE/L) & $1.22 \pm 0.54$ & $<0.001$ & $1.28 \pm 0.56$ & $1.17 \pm 0.51$ & 0.17 \\
\hline WWF:Ag HUT (kIE/L) & $1.28 \pm 0.55$ & & $1.33 \pm 0.59$ & $1.24 \pm 0.52$ & 0.31 \\
\hline WWF:GP1bA supine (kIE/L) & $1.24 \pm 0.55$ & 0.20 & $1.30 \pm 0.59$ & $1.19 \pm 0.52$ & 0.19 \\
\hline WWF:GP1bA HUT (kIE/L) & $1.26 \pm 0.56$ & & $1.31 \pm 0.64$ & $1.23 \pm 0.49$ & 0.34 \\
\hline Fibrinogen supine $(\mathrm{g} / \mathrm{L})$ & $2.75 \pm 0.60$ & $<0.001$ & $2.67 \pm 0.57$ & $2.81 \pm 0.62$ & 0.11 \\
\hline Fibrinogen HUT (g/L) & $2.84 \pm 0.60$ & & $2.73 \pm 0.59$ & $2.93 \pm 0.60$ & 0.022 \\
\hline LA1 supine (sec) & $31.2 \pm 3.7$ & 0.066 & $31.9 \pm 3.50$ & $30.7 \pm 3.8$ & 0.027 \\
\hline LA1 HUT (sec) & $31.1 \pm 4.7$ & & $32.0 \pm 3.92$ & $30.5 \pm 5.2$ & 0.030 \\
\hline APC-resistance supine & $2.60 \pm 0.41$ & $<0.001$ & $2.65 \pm 0.39$ & $2.57 \pm 0.42$ & 0.17 \\
\hline APC-resistance HUT & $2.42 \pm 0.43$ & & $2.49 \pm 0.41$ & $2.36 \pm 0.44$ & 0.044 \\
\hline APTT/C+ supine (sec) & $84.3 \pm 19.6$ & $<0.001$ & $85.8 \pm 19.8$ & $83.1 \pm 19.5$ & 0.36 \\
\hline $\mathrm{APTT} / \mathrm{C}+\mathrm{HUT}$ (sec) & $75.1 \pm 18.8$ & & $77.4 \pm 18.7$ & $73.1 \pm 18.7$ & 0.13 \\
\hline APTT/C- supine (sec) & $32.1 \pm 3.8$ & $<0.001$ & $32.1 \pm 4.1$ & $32.1 \pm 3.6$ & 1.0 \\
\hline APTT/C- HUT (sec) & $30.8 \pm 3.7$ & & $30.8 \pm 3.8$ & $30.7 \pm 3.7$ & 0.83 \\
\hline
\end{tabular}

*supine vs. HUT paired samples t-test; १ men vs. women independent samples t-test; FVIII, factor VIII; VWF:Ag, von Willebrand factor antigen; vVWF:GP1bA, von Willebrand factor GP1bA activity; LA1, lupus anticoagulant-screening; APC-resistance, activated protein C resistance; APTT, activated prothrombin time; $\mathrm{C}+/-$, with/without activated protein $\mathrm{C}$

Furthermore, we observed elevated levels of VWF during orthostasis. It has been reported that higher levels of VWF are associated with a 3-fold increased risk for severe coronary heart disease [28-30]. Our results are consistent with other studies, linking changes in VWF and FVIII to the vasovagal reflex, artificially induced orthostatic stress and presyncope $[5,6,8]$. The von Willebrand factor is the largest plasma protein and plays a key role in primary haemostasis as cofactor in

Table 3 Gender-stratified changes in coagulation markers during head-up tilt among 178 patients with unexplained syncope

\begin{tabular}{lcccc}
\hline & Men & $p$-value* & Women & $p$-value* \\
\hline $\mathrm{N}(\%)$ & $81(46)$ & & $97(54)$ & \\
$\Delta \mathrm{FVIII:C} \mathrm{(kIE/L)}$ & $0.00 \pm 0.16$ & 0.64 & $0.02 \pm 0.13$ & 0.091 \\
$\Delta$ WWF:Ag (kIE/L) & $0.04 \pm 0.18$ & 0.030 & $0.07 \pm 0.15$ & $<0.001$ \\
$\Delta$ WWF:GP1bA (kIE/L) & $0.01 \pm 0.25$ & 0.86 & $0.03 \pm 0.18$ & 0.067 \\
$\Delta$ Fibrinogen (g/L) & $0.06 \pm 0.32$ & 0.12 & $0.12 \pm 0.22$ & $<0.001$ \\
$\Delta$ LA1 (sec) & $-0.13 \pm 3.23$ & 0.72 & $-0.56 \pm 1.96$ & 0.007 \\
$\Delta$ APC-resistance & $-0.16 \pm 0.26$ & $<0.001$ & $-0.21 \pm 0.26$ & $<0.001$ \\
$\Delta$ APTT/C+ (sec) & $-8.3 \pm 13.7$ & $<0.001$ & $-9.9 \pm 10.6$ & $<0.001$ \\
$\Delta$ APTT/C- (sec) & $-1.2 \pm 2.4$ & $<0.001$ & $-1.4 \pm 1.7$ & $<0.001$
\end{tabular}

*supine vs. HUT paired samples t-test; FVIII, factor VIII; VWF:Ag, von Willebrand factor antigen; vWF:GP1bA, von Willebrand factor GP1bA activity; LA1, lupus anticoagulant-screening; APC-resistance, activated protein $\mathrm{C}$ resistance; APTT, activated prothrombin time; $\mathrm{C}+/-$, with/without activated protein $\mathrm{C}$ platelet adhesion and platelet aggregation. This platelet adhesion is promoted by a platelet membrane receptor glycoprotein (GPIb -IX-V) when circulating VWF attaches to the sub-endothelium collagen and serves as a bridge between tissue and platelets [31].

Previous studies performed on healthy subjects have shown that platelet aggregability is increased in the morning, when the incidence of cardiovascular events such as myocardial infarct, sudden cardiac death and transient myocardial ischemia is higher [1,32-35]. However, data on the changes in haemostatic plasma components in the same settings are very sparse and we believe that this study may contribute to the understanding of cardiovascular chronobiology. Our results confirm that changing from supine to upright body position induces activation of the coagulation system and promotes a hypercoagulable state during early orthostasis. The physiological role of orthostatic hypercoagulability may be to protect the body against increased bleeding risk during activities of daily life or be a result of higher hydrostatic pressure in the lower body, which will activate the endothelial response and release of coagulation factors.

The activation of haemostatic plasma components may support the hypothesis of tight cooperation between procoagulatory factors and platelets, and its impact on the development of cardiovascular events. Whether or not cardiovascular events such as myocardial infarct, sudden cardiac death, transient myocardial ischemia, 
Table 4 Age-stratified changes in coagulation markers during head-up tilt among 178 patients with unexplained syncope

\begin{tabular}{|c|c|c|c|c|}
\hline & Age $<65$ years & $p$-value* & Age $\geq 65$ years & $p$-value \\
\hline N (\%) & $119(67)$ & & $59(33)$ & \\
\hline$\Delta \mathrm{FVIII:C} \mathrm{(kIE/L)}$ & $0.02 \pm 0.15$ & 0.17 & $-0.01 \pm 0.15$ & 0.55 \\
\hline$\Delta$ WWF:Ag (kIE/L) & $0.04 \pm 0.15$ & 0.006 & $0.10 \pm 0.19$ & $<0.001$ \\
\hline$\Delta \mathrm{WWF}: \mathrm{GP} 1 \mathrm{bA}(\mathrm{kIE} / \mathrm{L})$ & $0.02 \pm 0.22$ & 0.35 & $0.02 \pm 0.19$ & 0.37 \\
\hline$\Delta$ Fibrinogen $(\mathrm{g} / \mathrm{L})$ & $0.10 \pm 0.30$ & $<0.001$ & $0.07 \pm 0.20$ & 0.009 \\
\hline$\Delta L^{\prime} 1$ (sec) & $-0.40 \pm 2.88$ & 0.14 & $-0.31 \pm 1.92$ & 0.25 \\
\hline$\triangle \mathrm{APC}$-resistance & $-0.16 \pm 0.24$ & $<0.001$ & $-0.24 \pm 0.28$ & $<0.001$ \\
\hline$\Delta \mathrm{APTT} / \mathrm{C}+(\mathrm{sec})$ & $-8.5 \pm 12.2$ & $<0.001$ & $-10.6 \pm 11.9$ & $<0.001$ \\
\hline$\Delta \mathrm{APTT} / \mathrm{C}-(\mathrm{sec})$ & $-1.3 \pm 2.1$ & $<0.001$ & $-1.3 \pm 2.0$ & $<0.001$ \\
\hline
\end{tabular}

*supine vs. HUT paired samples t-test; FVIII, factor VIII; VWF:Ag, von Willebrand factor antigen; vWF:GP1bA, von Willebrand factor GP1bA activity; LA1, lupus anticoagulant-screening; APC-resistance, activated protein C resistance; APTT, activated prothrombin time; $\mathrm{C}+/-$, with/without activated protein $\mathrm{C}$

and stroke during morning hours are promoted by synergic effects of both orthostatic hypercoagulability and increased platelet aggregability remains to be further explored.

Finally, patients diagnosed with vasovagal reflex syncope (VVS) demonstrated less pronounced changes in vWF:Ag than the rest of study population, whereas those with $\mathrm{OH}$ did not differ from non-OH patients. The former may be due to relatively younger age of VVS patients, although this study does not allow further speculations on the cause of this difference, whereas the latter implies that procoagulatory orthostatic changes are independent of BP fluctuations on standing.

\section{Limitations}

The conclusions of our study may be underpowered due to some important limitations (listed below). Thus, we would like to emphasize that the current study may be useful as a "proof of concept" for a larger and improved study design, in which these limitations could be overcome. No control group including healthy individuals was included and there were no measurements of coagulation parameters in subjects that did not undergo HUT. As the participants of the current study are part of the ongoing larger SYSTEMA project [11], no prospective sample size assessment was done, prior to the study. We were not able to measure specific markers of activated coagulation, such as thrombin-antithrombin-complex (TAT) or D-dimer, which would have been informative. Furthermore, since data on hereditary thrombophilia and levels or function of platelets were not registered, it was not feasible to separate possible contributions of these variables to hypercoagulability. Procoagulants were measured after 3 min of HUT only and we do not have data on how the prolonged standing might influence the assessed coagulation factors. However, determination of early orthostatic changes may have precluded the possible effects of intravascular volume escape observed during prolonged standing on the concentration of procoagulatory proteins. Also of relevance, even though the observed rise in coagulation markers is in direction during HUT, we cannot exclude a similar reaction if subjects were to be provoked by physical stress that did not involve orthostasis (such as on a supine ergometer bicycle). Finally, our study design and the fact that we did not measure potentially important coagulation proteases such as thrombin, factor $\mathrm{Xa}$ and tissue factor involved in atherosclerosis development, precludes us from drawing any conclusions on whether syncope induced hypercoagulability might also contribute to the development of atherosclerosis.

\section{Conclusions}

Procoagulatory changes in haemostatic plasma components can be observed during early phase of passive orthostatic challenge irrespective of syncope diagnosis. These findings may contribute to the understanding of orthostatic hypercoagulability and chronobiology of cardiovascular disease.

\section{Abbreviations \\ APC-resistance: Activated protein C resistance; APTT: Activated partial thromboplastin time; FVIII:C: Factor VIII activity; HUT: Head-up tilt test; LA1: Lupus anticoagulant; $\mathrm{OH}$ : Orthostatic hypotension; WS: Vasovagal syncope; WWF:Ag: Antigen concentration of von Willebrand factor; WWF:GP1bA: Activity of von Willebrand factor}

\section{Acknowledgments}

Authors would like to thank Philippe Burri, Amna Ali, Elisabeth Persson and Shakila Modaber for their support and engagement during this study.

\section{Funding}

This study was supported by grants from the European Research Council (StG 282,225), Swedish Medical Research Council, the Swedish Heart and Lung Foundation, the Medical Faculty of Lund University, the Crafoord Foundation, the Ernhold Lundströms Research Foundation, the Hulda and Conrad Mossfelt Foundation, and the Anna Lisa and Sven-Erik Lundgrens Foundation. 


\section{Availability of data and materials}

The datasets used and/or analysed during the current study available from the corresponding author on reasonable request.

\section{Authors' contributions}

AF preformed all the examinations. VH, AF and NI performed statistical analyses and drafted the manuscript. KS and RS, added specific sections of manuscript (coagulation analyses, interpretation of results, and discussion). All authors contributed equally to critical review of manuscript and final editing. AF supported the work by grants. All authors read and approved the final manuscript.

\section{Competing interests}

The authors declare that they have no competing interests.

\section{Consent for publication}

Not applicable.

\section{Ethics approval and consent to participate}

The Regional Ethical Review Board in Lund, Sweden accepted the study protocol (ref no 82/2008), and all study participants gave their written informed consent.

\section{Publisher's Note}

Springer Nature remains neutral with regard to jurisdictional claims in published maps and institutional affiliations.

\section{Author details \\ 'Department of Clinical Sciences Malmö, Lund University, SE 205-02 Malmö, Sweden. ${ }^{2}$ Department of Internal Medicine, Skåne University Hospital, SE 205-02 Malmö, Sweden. ${ }^{3}$ Department of Medical Imaging and Physiology, Skåne University Hospital, SE 205-02 Malmö, Sweden. ${ }^{4}$ Department of Cardiology, Skåne University Hospital, Inga Marie Nilssons gata 46, SE 205-02 Malmö, Sweden. ${ }^{5}$ Centre for Thrombosis and Haemostasis, Skåne University Hospital, SE 205-02 Malmö, Sweden. ${ }^{6}$ National Heart and Lung Institute, Imperial College, Hammersmith Hospital Campus, Ducane Road, London W12 0NN, UK. ${ }^{7}$ Department of Cardiology, Skåne University Hospital, SE 221-85 Lund, Sweden.}

\section{Received: 30 March 2017 Accepted: 13 June 2017}

\section{Published online: 20 June 2017}

\section{References}

1. Tofler GH, Brezinski D, Schafer Al, Czeisler CA, Rutherford JD, Willich SN, et al. Concurrent morning increase in platelet aggregability and the risk of myocardial infarction and sudden cardiac death. N Engl J Med. 1987; 316(24):1514-8

2. Goldberg RJ, Brady P, Muller JE, Chen ZY, de Groot M, Zonneveld P, et al. Time of onset of symptoms of acute myocardial infarction. Am J Cardiol. 1990;66(2):140-4.

3. Gallerani M, Manfredini R, Ricci L, Grandi E, Cappato R, Calo G, et al. Sudden death from pulmonary thromboembolism: chronobiological aspects. Eur Heart J. 1992;13(5):661-5.

4. Elliott WJ. Circadian variation in the timing of stroke onset: a meta-analysis. Stroke. 1998;29(5):992-6.

5. Masoud M, Sarig G, Brenner B, Jacob G. Orthostatic hypercoagulability: a novel physiological mechanism to activate the coagulation system. Hypertension. 2008;51(6):1545-51.

6. Masoud M, Sarig G, Brenner B, Jacob G. Hydration does not prevent orthostatic hypercoagulability. Thromb Haemost. 2010;103(2):284-90.

7. Cvirn G, Schlagenhauf A, Leschnik B, Koestenberger M, Roessler A, Jantscher $A$, et al. Coagulation changes during presyncope and recovery. PLoS One. 2012;7(8):e42221.

8. Kraemer M, Kuepper M, Nebe-vom Stein A, Sorgenfrei U, Diehl RR. The influence of vasovagal response on the coagulation system. Clin Auton Res. 2010;20(2):105-11.

9. Casonato A, Pontara E, Bertomoro A, Cattini MG, Soldera C, Girolami A. Fainting induces an acute increase in the concentration of plasma factor VIII and von Willebrand factor. Haematologica. 2003;88(6):688-93.
10. Isma N, Sutton R, Hillarp A, Strandberg K, Melander O, Fedorowski A. Higher levels of von Willebrand factor in patients with syncope due to orthostatic hypotension. J Hypertens. 2015;33(8):1594-601.

11. Fedorowski A, Burri $\mathrm{P}$, Juul-Moller S, Melander O. A dedicated investigation unit improves management of syncopal attacks (syncope study of unselected population in Malmo-SYSTEMA I). Europace. 2010;12(9):1322-8.

12. Bartoletti A, Alboni P, Ammirati F, Brignole M, Del Rosso A, Foglia Manzillo G, Menozzi C, Raviele A, Sutton R. 'The Italian Protocol': a simplified head-up tilt testing potentiated with oral nitroglycerin to assess patients with unexplained syncope. Europace. 2000;2(4):339-42.

13. Eeftinck Schattenkerk DW, van Lieshout JJ, van den Meiracker $A H$ Wesseling KR, Blanc S, Wieling W, et al. Nexfin noninvasive continuous blood pressure validated against Riva-Rocci/Korotkoff. Am J Hypertens. 2009;22(4):378-83.

14. Nilsson D, Sutton R, Tas W, Burri P, Melander O, Fedorowski A. Orthostatic changes in Hemodynamics and cardiovascular biomarkers in Dysautonomic patients. PLoS One. 2015:10(6):e0128962.

15. Moya A, Sutton R, Ammirati F, Blanc JJ, Brignole M, Dahm JB, et al. Guidelines for the diagnosis and Management of Syncope (version 2009): the task force for the diagnosis and management of syncope of the European Society of Cardiology (ESC). Eur Heart J. 2009:30(21):2631-71.

16. Meade TW, Mellows S, Brozovic M, Miller GJ, Chakrabarti RR, North WR, et al. Haemostatic function and ischaemic heart disease: principal results of the Northwick Park heart study. Lancet. 1986;2(8506):533-7.

17. Kannel WB, Wolf PA, Castelli WP, D'Agostino RB. Fibrinogen and risk of cardiovascular disease. The Framingham study. JAMA. 1987;258(9):1183-6.

18. Yarnell JW, Baker IA, Sweetnam PM, Bainton D, O'Brien JR, Whitehead PJ, et al. Fibrinogen, viscosity, and white blood cell count are major risk factors for ischemic heart disease. The Caerphilly and speedwell collaborative heart disease studies. Circulation. 1991;83(3):836-44.

19. Stec JJ, Silbershatz H, Tofler GH, Matheney TH, Sutherland P, Lipinska I, et al. Association of fibrinogen with cardiovascular risk factors and cardiovascular disease in the Framingham offspring population. Circulation. 2000;102(14):1634-8.

20. Smith EB, Keen GA, Grant A, Stirk C. Fate of fibrinogen in human arterial intima. Arteriosclerosis. 1990;10(2):263-75.

21. Blomback M, Konkle BA, Manco-Johnson MJ, Bremme K, Hellgren M, Kaaja R. Issues ISSOWsH: Preanalytical conditions that affect coagulation testing, including hormonal status and therapy. J Thromb Haemost. 2007;5(4):855-8.

22. Group ECW. Venous thromboembolism in women: a specific reproductive health risk. Hum Reprod Update. 2013;19(5):471-82.

23. $\mathrm{Ng} \mathrm{VL}$. Prothrombin time and partial thromboplastin time assay considerations. Clin Lab Med. 2009;29(2):253-63.

24. Korte W, Clarke S, Lefkowitz JB. Short activated partial thromboplastin times are related to increased thrombin generation and an increased risk for thromboembolism. Am J Clin Pathol. 2000;113(1):123-7.

25. Mina A, Favaloro EJ, Mohammed S, Koutts J. A laboratory evaluation into the short activated partial thromboplastin time. Blood Coagul Fibrinolysis. 2010;21(2):152-7.

26. Pinelli A, Trivulzio S, Rossoni G. Activated partial thromboplastin time correlates with methoxyhydroxyphenylglycol in acute myocardial infarction patients: therapeutic implications for patients at cardiovascular risk. In Vivo. 2014:28(1):99-104.

27. Abdullah WZ, Moufak SK, Yusof Z, Mohamad MS, Kamarul IM. Shortened activated partial thromboplastin time, a hemostatic marker for hypercoagulable state during acute coronary event. Transl Res. 2010;155(6):315-9.

28. Whincup PH, Danesh J, Walker M, Lennon L, Thomson A, Appleby P, Rumley A, Lowe GD. von Willebrand factor and coronary heart disease: prospective study and meta-analysis. Eur Heart J. 2002;23(22):1764-70.

29. Willeit $P$, Thompson A, Aspelund T, Rumley A, Eiriksdottir G, Lowe G, et al. Hemostatic factors and risk of coronary heart disease in general populations: new prospective study and updated meta-analyses. PLoS One. 2013:8(2):e55175.

30. Morange PE, Simon C, Alessi MC, LuC G, Arveiler D, Ferrieres J, et al. Endothelial cell markers and the risk of coronary heart disease: the prospective epidemiological study of myocardial infarction (PRIME) study. Circulation. 2004;109(11):1343-8.

31. Berndt MC, Shen Y, Dopheide SM, Gardiner EE, Andrews RK. The vascular biology of the glycoprotein Ib-IX-V complex. Thromb Haemost. $2001 ; 86(1): 178-88$. 
32. Brezinski DA, Tofler GH, Muller JE, Pohjola-Sintonen S, Willich SN, Schafer Al, et al. Morning increase in platelet aggregability. Association with assumption of the upright posture. Circulation. 1988;78(1):35-40.

33. Willich SN, Tofler GH, Brezinski DA, Schafer Al, Muller JE, Michel T, et al.

Platelet alpha 2 adrenoceptor characteristics during the morning increase in platelet aggregability. Eur Heart J. 1992;13(4):550-5.

34. Muller JE. Morning increase of onset of myocardial infarction. Implications concerning triggering events. Cardiology. 1989;76(2):96-104.

35. Muller JE. Circadian variation and triggering of acute coronary events. Am Heart J. 1999;137(4 Pt 2):S1-8.

Submit your next manuscript to BioMed Central and we will help you at every step:

- We accept pre-submission inquiries

- Our selector tool helps you to find the most relevant journal

- We provide round the clock customer support

- Convenient online submission

- Thorough peer review

- Inclusion in PubMed and all major indexing services

- Maximum visibility for your research

Submit your manuscript at www.biomedcentral.com/submit 